\title{
Others' emotions teach, but not in autism: an eye-tracking pupillometry study
}

Heather J. Nuske ${ }^{1,2^{*}}$, Giacomo Vivanti ${ }^{2,3}$ and Cheryl Dissanayake ${ }^{2}$

\begin{abstract}
Background: Much research has investigated deficit in emotional reactivity to others in people with autism, but scant attention has been paid to how this deficit affects their own reactions to features of their environment (objects, events, practices, etc.). The present study presents a preliminary analysis on whether calibrating one's own emotional reactions to others' emotional reactions about features of the world, a process we term social-emotional calibration, is disrupted in autism.

Methods: To examine this process, we used a novel eye-tracking pupillometry paradigm in which we showed 20 preschoolers with autism and 20 matched typically developing preschoolers' videos of an actor opening a box and reacting to the occluded object inside, with fear or happiness. We expected preschoolers to come to perceive the box as containing a positive or threatening stimulus through emotionally calibrating to the actor's emotional expressions. Children's mean pupil diameter (indicating emotional reactivity) was measured whilst viewing an up-close, visually identical image of the box before and then after the scene, and this difference was taken as an index of social-emotional calibration and compared between groups.

Results: Whilst the typically developing preschoolers responded more emotionally to the box after, compared to before the scene (as indexed by an increase in pupil size), those with autism did not, suggesting their reaction to the object was not affected by the actor's emotional expressions. The groups did not differ in looking duration to the emotional expressions; thus, the pupil dilation findings cannot be explained by differences in visual attention. More social-emotional calibration on the happy condition was associated with less severe autism symptoms.

Conclusions: Through the measurement of physiological reactivity, findings suggest social-emotional calibration is diminished in children with autism, with calibration to others' positive emotions as particularly important. This study highlights a possible mechanism by which individuals with autism develop idiosyncratic reactions to features of their environment, which is likely to impact their active and harmonious participation on social and cultural practices from infancy, throughout the lifespan. More research is needed to examine the mediators and developmental sequence of this tendency to emotionally calibrate to others' feelings about the world.
\end{abstract}

Keywords: Social-emotional calibration, Autism, Emotion, Eye-tracking pupillometry, Autonomic nervous system, Social learning

\footnotetext{
* Correspondence: hjnuske@upenn.edu
${ }^{1}$ Center for Mental Health Policy and Services Research, Perelman School of Medicine, Department of Psychiatry, University of Pennsylvania, Philadelphia, PA 19104, USA

${ }^{2}$ Olga Tennison Autism Research Centre, School of Psychological Science, La

Trobe University, Melbourne, Victoria 3086, Australia

Full list of author information is available at the end of the article
} 


\section{Background}

People with Autism Spectrum Disorder (ASD), a neurodevelopmental disorder characterised by impairments in social communication and behavioural rigidity [1], often present with atypical, reduced or delayed reactions to others' emotions [2, 3]. They also frequently present with idiosyncratic, extreme or otherwise unusual emotional reactions to particular features in their physical environment (objects, sensations, events, etc.) [4-7]. Though these phenomena are often documented in ASD, the link between atypical emotional responses to people and atypical emotional responses to non-social environmental features is not clear. In particular, no study to date has investigated whether observing another person reacting emotionally to features of the world influences how individuals with ASD react to those features, for example, how a teacher smiling while reading a book influences the reaction of a boy with autism to that book.

We know that emotional expressions of significant others teach young typically developing children about the world around them, without the need of explicit verbal instruction; by 12 months, children spontaneously seek out emotional feedback from their caregivers to determine appropriate behavioural adjustments in different contexts (i.e. they engage in social referencing; [8-12]). Acquiring knowledge about how people react to features of the world from others' emotions is an effective and efficient learning mechanism that children use prior to language onset and which continues to shape behaviour throughout the lifespan [13].

Preliminary research on such learning from others' emotions in ASD suggests difficulties in this area relative to their typically developing peers $[14,15]$, which is keeping with our current understanding of ASD as a disorder of social learning [16-18], i.e. learning from other people. However, given that reduced/atypical attention to social stimuli, such as faces, is a common feature of ASD [19, 20], it is unclear whether these difficulties are the consequence of not seeking out or paying attention to others' emotional expressions regarding features of their environment, or whether children with ASD do look but fail to calibrate their own emotional reactions to the reactions of others. We define this process, social-emotional calibration, a process by which, after observing or experiencing another's emotional expression (e.g. facial, vocal or bodily) in response to a particular referent (e.g. an object, event, topic, social or cultural practice, attitude or another person), the observer's emotional reactions to that referent calibrate with those of the observed person. It is proposed that children learn to react appropriately to objects and situations in their environment based on the observed emotions of others toward those objects or situations. For example, through seeing his mother laugh at a clown, a child can learn that the clown is not scary and join in laughing at it. Likewise, by observing the pleasure expressed on other children's faces at birthday parties, a child can be moved to enjoy the event and look forward to his upcoming birthday.

Like social referencing, social-emotional calibration is a triadic process between two people and a referent. However, whilst some previous work using social referencing paradigms has investigated the act of seeking out others' emotional reactions to guide behaviour, the current study focuses on the change in emotional reaction to a particular referent that occurs as a consequence of witnessing others' emotions to that referent. The foundation of this process, we argue, is rooted in early social attention and social-emotional processes including gaze following, emotion recognition and emotional contagion [21, 22]. Individuals with ASD have difficulties with each of these processes, including reduced attention to people and difficulties in recognising and implicitly responding to others' emotions and expressing their own emotions, leading to difficulties in empathic responding in this population [3]. A large body of research has investigated physiological and neurophysiological reactivity to others' emotions in ASD. Findings suggest atypical responses to both positive and negative emotions at both levels of implicit and explicit processing (e.g. [23-30]). Furthermore, slower responses to emotions have been reported across physiological and behavioural methodologies [31-35]. Therefore, we expected children with ASD to have reduced social-emotional calibration due to difficulties with processing others' emotions. We wanted to know whether children with ASD learn to react appropriately to objects and situations in their environment based on the observed emotions of others to those objects and situations.

To date, most of the research referred to above has investigating physiological and neurophysiological emotional reactivity has focused on older high-functioning children and adults due to the invasive and movementsensitive technology commonly used in these studies (event-related potentials (ERP), functional magnetic resonance imaging (fMRI), electrocardiogram (ECG)). Little is known about emotion processing in younger, more affected children, and the findings mentioned above may not apply to this population. Eye-tracking pupillometry is an ideal measure of physiological responses in this group as no electrodes are required for recording and this technology is more tolerant of movement than other physiological recording systems (e.g. systems for measuring skin conductance responses). Pupillometry measures pupil size over time; the pupils dilate in response to stimuli that are high in emotional intensity, regardless of valence (e.g. [36-39]). Pupil dilation has been found to be correlated with skin conductance responses whilst viewing emotional images [36], which is indicative of the 
role of sympathetic nervous system responses. Atypical pupil dilation to emotion-inducing stimuli has been found in clinical populations such as people with depression [40, 41] and post-traumatic stress disorder [42], as well as children at risk for depression and anxiety [43] and healthy adults who have been sleep-deprived [44]. Recently, this technology has been applied to individuals with ASD to look at responses to social vs. non-social stimuli in lower-functioning young children [45]. Since then, pupillometry has been used to examine a number of questions in ASD (and children at risk for ASD) including those related to resting-state physiology [46-48], sensory-processing $[49,50]$, eye-gaze processing [51] and general face processing [52]. Pupillometry findings on emotion processing in ASD are consistent with findings from on older, more-able children and adults, including finding reduced and delayed emotional responses in the population [24, 30, 53-55].

\section{The current study}

Our primary aim was to test the hypothesis that socialemotional calibration is disrupted in children with ASD, compared to typically developing children. Additionally, we wanted to understand whether difficulties in calibrating to others' emotions reflect a lack of attention to others' emotional expressions, or difficulties in socialemotional calibration per se, and whether ASD symptoms are associated with social-emotional calibration.

\section{Methods}

\section{Participants}

Twenty-six children with ASD and 24 typically developing (TD) children, aged 2 to 5 years, participated in the study. However, six children in the ASD group and four children in the TD group were excluded as they looked $<20 \%$ of the duration of one or both of the up-close images of the boxes shown in the video stimuli (pre-/postbox, see 'Materials' section), resulting in a total of 20 children in each group. This threshold was chosen as this allowed us to obtain a reasonable duration of minimum viewing time $(800 \mathrm{~ms})$ in order to determine the mean pupil diameter whilst viewing the boxes. Participant characteristics are presented in Table 1. Both groups were recruited through the same community childcare centre offering services for children with ASD and TD children. The Mullen Scales of Early Learning (MSEL; [56]) was administered to all participants to measure cognitive ability. As expected, the ASD group was lower in cognitive ability than the TD group. Cognitive ability was entered as a covariate in the analysis. Following the recommendations of Dykens and Lense [57], the sample included low- to high-functioning children with ASD [58], with $65 \%$ low functioning (standard
Table 1 Participant characteristics

\begin{tabular}{llll}
\hline & $\begin{array}{l}\text { ASD group } \\
(N=20)\end{array}$ & $\begin{array}{l}\text { TD group } \\
(N=20)\end{array}$ & $\begin{array}{l}\text { Comparison } \\
\text { coefficients }\end{array}$ \\
\hline Age (years): $M(S D)$ & $4.03(1.09)$ & $4.25(.63)$ & $\begin{array}{l}t(38)=.75, \\
p=.46\end{array}$ \\
Gender: M, F & 16,4 & 16,4 & - \\
MSEL, SS: $M(S D)^{a}$ & $69.50(23.10)$ & $102.00(17.84)$ & $t(38)=4.98$, \\
& & & - \\
ADOS, C-SevSc: $M(S D)^{b}$ & $6.45(2.44)$ & - & - \\
ADOS, C-SA: $M(S D)^{c}$ & $11.80(4.14)$ & - & - \\
ADOS, C-RRB: $M(S D)^{d}$ & $4.40(2.14)$ & - & - \\
\hline
\end{tabular}

${ }^{a}$ Mullen Scales of Early Learning Standard Score (Early Learning Composite)

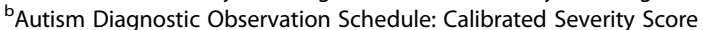

'Autism Diagnostic Observation Schedule: Calibrated Social Affect Score

${ }^{d}$ Autism Diagnostic Observation Schedule: Calibrated Restricted and Repetitive Behaviours Score

score $<70$ ), $10 \%$ moderately functioning (standard score $70-84$ ) and $25 \%$ high functioning (standard score $\geq 85$ ).

Clinic-based diagnoses of the children with ASD were confirmed using the Autism Diagnostic Observation Schedule [59], by clinicians certified for research purposes on the administration and coding of the Autism Diagnostic Observation Schedule (ADOS), with 14 children meeting the more strict cut-off for autistic disorder and 6 meeting criteria for ASD. The ADOS scores were converted to ADOS calibrated severity scores using the algorithms provided by Gotham et al. [60]. One participant was taking methylphenidate at the time of testing. However, as this participant was not an outlier on any dependent variable, and given that results remained unchanged with the exclusion of his data, he was retained in the sample. All participants were free from any other medical conditions, and had no visual, hearing or motor impairments. The research was approved by the La Trobe University Human Ethics Committee (approval number 11-052), and consent from the children's parents was obtained according to the Declaration of Helsinki (BMJ 1991; 302: 1194).

\section{Apparatus}

A Tobii 120 binocular eye tracker and Tobii Studio software (version 3.0.3 Tobii, Stockholm, Sweden) were used to present stimuli and record visual attention and pupil diameter. This system presents stimuli on a computerlike monitor and does not require any equipment to be fastened onto the participant. Using multiple sensors, with bright and dark pupil tracking, a 3D model of the pupil (taking into account optical distortions from the cornea and lens) is built, allowing for both pupil diameter and distance from the screen to be measured at a sampling rate of $60 \mathrm{~Hz}$ (one sample every $16.67 \mathrm{~ms}$ ). With this tracking technique, movement-related artefacts are handled in two ways. Firstly, as pupil diameter is a function of distance from the screen (of participant's 
head to the monitor), the effect of head movements perpendicular to the monitor are eliminated from the measure of pupil size on a sample-to-sample basis, using basic principles of trigonometry. Secondly, other head movements (i.e. those parallel to the monitor) are accurately tracked (up to $25 \mathrm{~cm}$ per second). Artefacts due to partial head turns and blinks were removed through data reduction algorithms (see 'Data reduction' section). The eye-tracking monitor (TFT-LCD; W: $34 \mathrm{~cm} \times \mathrm{H}: 27 \mathrm{~cm}$ ) has a refresh rate of $60 \mathrm{~Hz}$. Brightness was set to $100 \%$.

\section{Materials}

There were two study conditions: the happy condition and the fear condition. One video was shown for each of the conditions. Each featured a woman opening a plain box and reacting emotionally (either happily or fearfully) to the occluded object inside. An up-close image of the box was shown before and after the scene; during these two stimuli, the pupil diameter measure was taken (see Fig. 1 for a summary of the video sections).

First, a scrambled image of the close-up of the box was shown to minimise pre-stimuli to stimulus changes in luminosity (1 s). Second, a close-up still image of the box was shown, the 'pre-box' (4 s), before the actor sequence. Third, the video frame zooms from the close-up image of the pre-box to the upper torso of the actor sitting at a table with a neutral face and averted eye gaze, still showing the box in the corner of the frame (happy condition: $7.60 \mathrm{~s}$, fear condition: $8.23 \mathrm{~s}$ ). Fourth, the actor looks toward the camera with a neutral expression, then looks at the box and moves her arms to open it (happy condition: $5.93 \mathrm{~s}$, fear condition: $5.83 \mathrm{~s}$ ). Fifth, the actor opens the box and reacts emotionally (happily or fearfully) to the contents of the box, which cannot be seen by the participant. Sixth, the actor closes the box and looks back at the camera, retaining her emotional expression. In total, the emotional expression of the actor is shown for $6.78 \mathrm{~s}$ in the happy condition and $6.99 \mathrm{~s}$ in the fear condition. Seventh, the camera moves away from the actor's face and slowly zooms back to a close-up of the box (4.097 $\mathrm{s}$ in the happy condition and $4.107 \mathrm{~s}$ in the fear condition), to allow enough time for resolution of the pupil dilation associated with viewing an emotional facial expression [53]. Finally, the box is shown again, the 'post-box' (4 s). In each of the videos, the still image of the post-box was visually identical to the pre-box. The total video length was 33.41 in the happy condition and 34.15 in the fear condition.

We reasoned that participants would perceive the prebox as an emotionally neutral stimulus and the post-box as a threatening stimulus (in the fear condition) or a positive stimulus (in the happy condition), as a consequence of calibrating their emotional reactions about the box to those of the actor. Following this line of reasoning, if participants failed to either look or calibrate to the actor's emotions, then they would continue to perceive the box as a neutral stimulus. The process of change from perceiving the box as a neutral stimulus to perceiving it as an emotionally relevant stimulus was measured by pupil size increase, an index of emotional reactivity. A larger pupil size during viewing of the postbox (an emotionally conditioned stimulus) compared to pre-box (a neutral stimulus) was used as an index of social-emotional calibration.

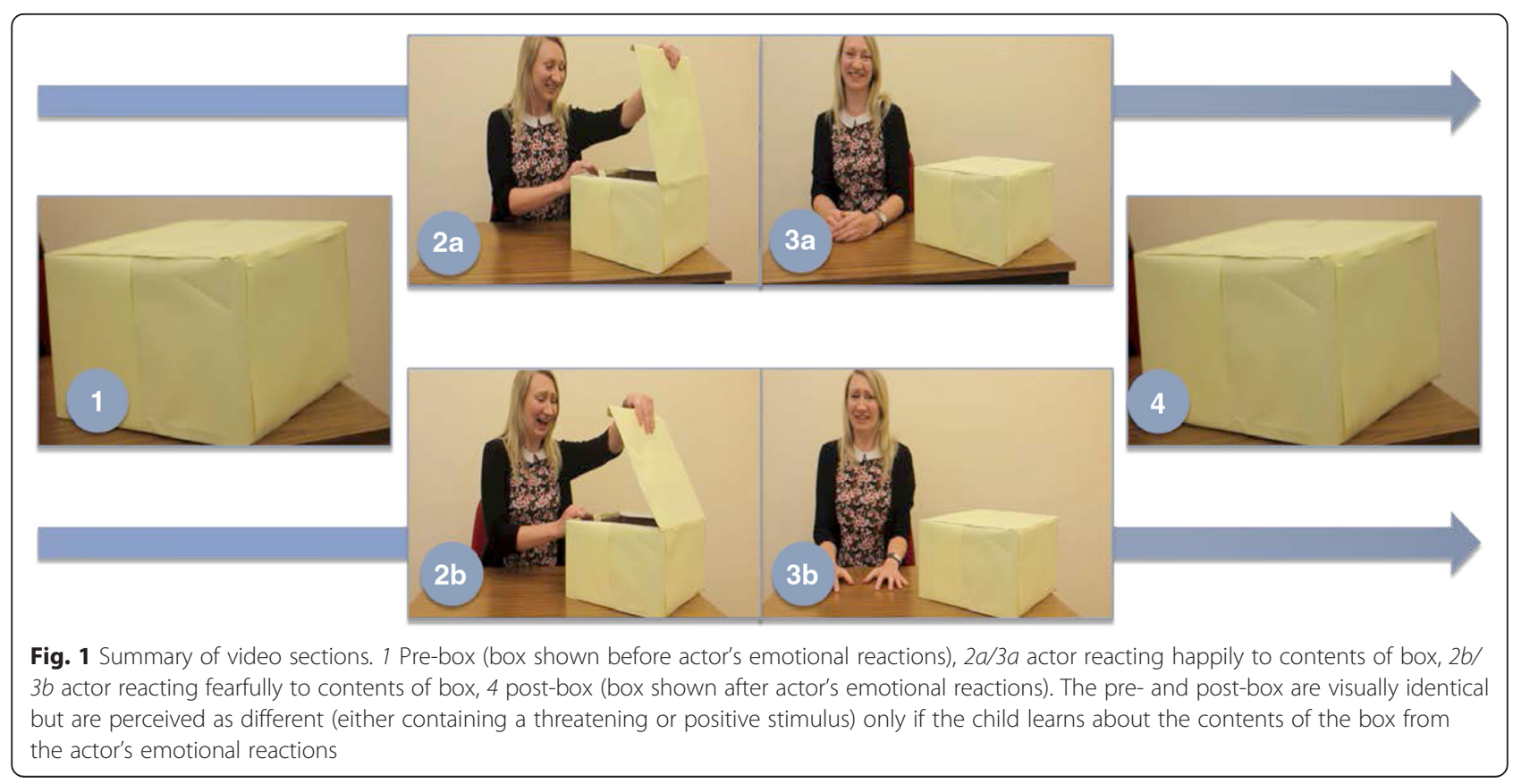




\section{Procedure}

Testing took place in a well-lit room of the community childcare centre which had no external light. Ambient luminosity was checked prior to each testing session, using a handheld photometer (model PLMX, Quantam Instruments). Ambient luminosity (lux) did not differ during testing between the ASD group $(M=25.630, \mathrm{SD}=$ $.156)$ and TD group $(M=25.628, \mathrm{SD}=.200), t(36)=-.038$, $p=.970$. The child was seated in a comfortable chair, approximately $60 \mathrm{~cm}\left(36.46^{\circ}\right.$ visual angle $)$ from the eye-tracking monitor. The experimenter first calibrated the child's eye movements with the built-in five-point Tobii Studio calibration procedure. Following this, each child passively viewed the images (with the emotion shown in the first video counterbalanced within each participant group), which were interspersed between the presentation of 'filler' stimuli (child-friendly pictures) to maintain attention [61]. The experiment presented here was a part of a larger study examining emotional responses in children with ASD (see [30, 46, 51, 53]).

\section{Data reduction}

Pupil data, preprocessed to minimise large movement artefacts (see 'Apparatus' section), were further processed with a custom-built LabVIEW 2010 (National Instruments, Austin, Texas, USA) algorithm (Beaton, unpublished), based on previously published methodology (e.g. $[62,63])$ to further screen out movement-related artefacts (including partial head turns and blinks). First, samples for which only one eye was tracked were eliminated (to minimise pupil size miscalculation due to head angle or ambient light exposure). Where both eyes were tracked, a mean pupil diameter across eyes was computed. Second, to remove extreme sample-to-sample changes in pupil diameter due to partial eyelid closures (common in samples either side of missing data due to blinks), samples outside $2 \times$ standard deviations of the mean rate of change (calculated for each participant) were removed. After partial head turn- and blink-related artefacts were deleted, missing pupil data rates were calculated by group (pre-interpolation, whole video): happy condition: ASD group range $=1-70 \%, M=34 \%, \mathrm{SD}=$ $21 \%$, TD group range $=2-72 \%, M=24 \%, \mathrm{SD}=20 \%$; fear condition: ASD group range $=2-77 \%, M=41 \%$, $\mathrm{SD}=25 \%$, TD group range $=2-48 \%, M=22 \%, \mathrm{SD}=$ $15 \%$. Third, gaps in data, due to blinks, were only linearly interpolated between stable data points (traces) to a maximum of $350 \mathrm{~ms}[64,65]$. A trace was deemed stable if there were a minimum of $50 \%$ of the samples in $2 \times$ total length of the gap, pre- and post-gap. This method allowed for a differential threshold for linear interpolation, based on gap length and the reliability of the pre/post-gap data.
A relative percentage change measure of pupil dilation (increase in size) was calculated using the last $300 \mathrm{~ms}$ of the $1 \mathrm{~s}$ scrambled image (to avoid the pupillary light reflex; [66]) which appeared directly before the onset of the pre-box, as a baseline. The following formula was used:

$$
a=(b-c) / c \times 100
$$

where $a$ is the percentage change from baseline to the following sections of the video: pre-box, emotional reaction, zoom in and post-box, $b$ is the mean pupil diameter during pre-box, emotional reaction, zoom in and post-box video sections and $c$ is the mean pupil diameter during the $300 \mathrm{~ms}$ before the onset of the pre-box (i.e. the last $300 \mathrm{~ms}$ of the scrambled image), per participant. To create a variable to represent social-emotional calibration we then subtracted the new relative pre-box variable from the post-box variable, where positive values equals more social-emotional calibration. As the pre- and post-box was visually identical, more pupil dilation in the post- vs. pre-box was taken as an index of learning about the happy- or fear-inducing contents of the box, through the actors' emotional expressions shown in the scene.

Two sets of areas of interest (AOIs) were created with the Tobii Studio software. The first was for the happy and fearful faces to measure visual attention to these facial expressions (see Fig. 2) and the second was to measure visual attention to the pre- and post-boxes (these were the size of the whole screen). Visual attention data (total fixation duration within the face AOIs) was also extracted from Tobii Studio using a fixation filter (I-VT), using the default pre-sets (maximum gap length $75 \mathrm{~ms}$, window length $20 \mathrm{~ms}$, velocity threshold 30 degrees per second, maximum time between fixations $75 \mathrm{~ms}$, maximum angle between fixations $.5^{\circ}$ ), with the exception that the minimum fixation duration was set to $100 \mathrm{~ms}$. This minimum fixation duration was chosen as eye-tracking data of $100 \mathrm{~ms}$ or more are not only more reliable than data tracked for shorter durations [67] but are also considered to be a reliable index of what elements in a scene are actually captured and processed [68].

\section{Results}

Data were first analysed for skewness, kurtosis and outliers using the method outlined in [69], with a critical value set at $+/-3.29$. Data were normally distributed (all critical values under 3.18); therefore, parametric tests were used in all analyses. As the index of socialemotional calibration (pre- to post-box pupil dilation) in the two emotion conditions (happy, fear) was not correlated in the TD group $(r=-09, p=.69)$, and only 

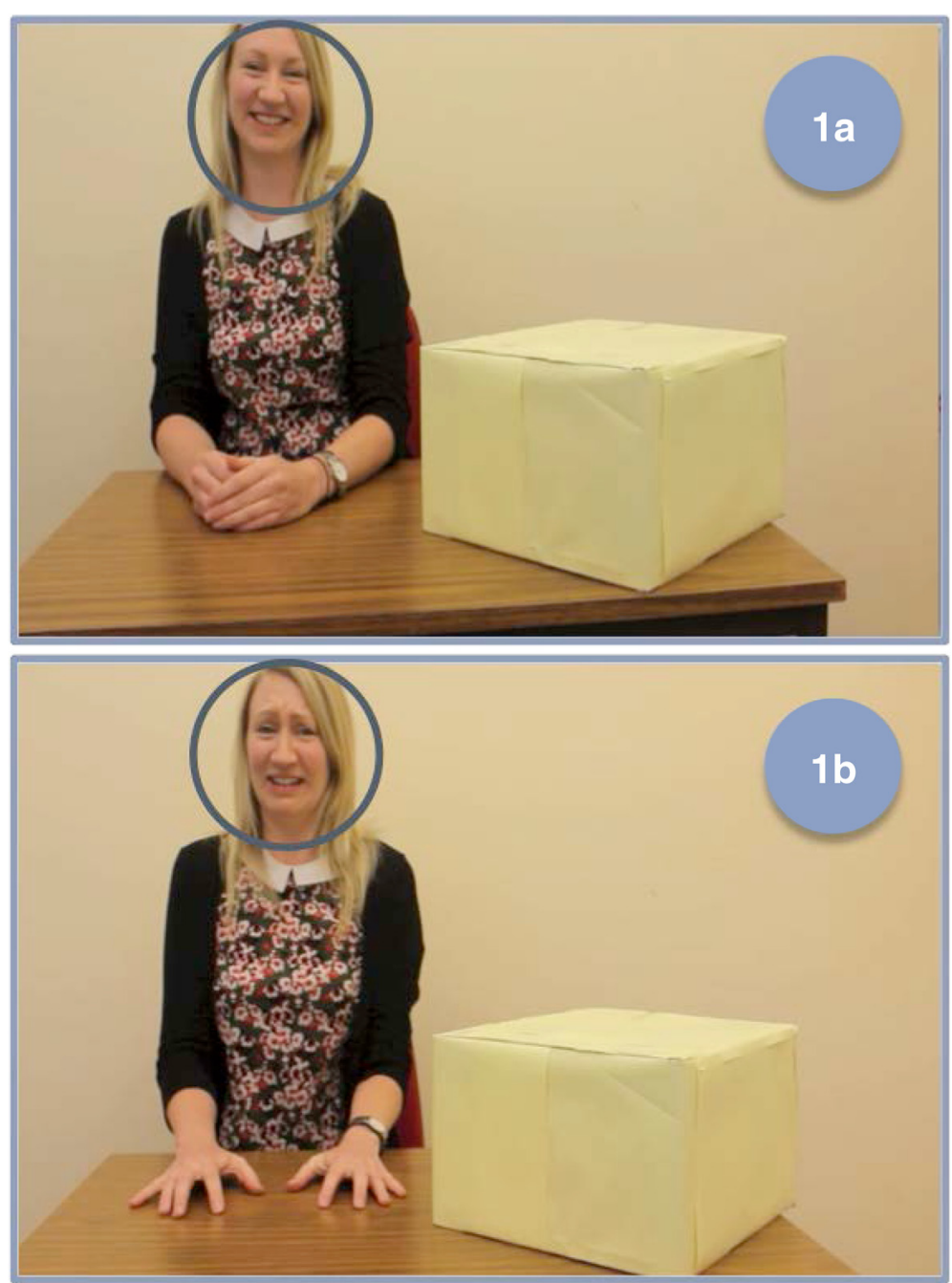

Fig. 2 Face areas of interest (AOls) for the happy (1a) and fear (1b) conditions. These AOls last the total time the emotional expressions are shown on the actor's face (happy condition $=6.78 \mathrm{~s}$, fear condition $=6.99 \mathrm{~s}$ ), from when she reacts after opening the box to before the camera zooms back into the box close-up

marginally correlated in the ASD group $(r=.45, p=.05)$, the happy and fear conditions were analysed separately.

\section{Visual attention to the pre- and post-boxes}

To determine whether the groups differed in their visual attention to the pre- and post-box images, independent sample $t$ tests were performed on the total fixation durations within the AOIs for the up-close images of the box which appeared before and after the actor scene $(4 \mathrm{~s}$ each). The groups differed in visual attention on the happy condition pre-box $(t(38)=2.66, p=.01)$ and the fear condition post-box $(t(38)=3.81, p<.001)$, but not the happy condition post-box $(t(38)=1.41, p=.17)$ or the fear condition pre-box $(t(38)=1.91, p=.06)$. However, both groups viewed each of the boxes long enough for a stable measure of pupil diameter [67, 68] (TD happy pre- and post-box: $M=2.62 \mathrm{~s}, \mathrm{SD}=.82 \mathrm{~s}$ and
$M=2.24 \mathrm{~s}, \mathrm{SD}=.58 \mathrm{~s}$, respectively; $\mathrm{ASD}$ happy preand post-box: $M=1.94 \mathrm{~s}, \mathrm{SD}=.81 \mathrm{~s}$ and $M=1.91 \mathrm{~s}$, $\mathrm{SD}=.86 \mathrm{~s}$, respectively; TD fear pre- and post-box: $M=2.52 \mathrm{~s}, \mathrm{SD}=.54 \mathrm{~s}$ and $M=2.76 \mathrm{~s}, \mathrm{SD}=.76 \mathrm{~s}$, respectively; ASD fear pre- and post-box: $M=2.11 \mathrm{~s}$, $\mathrm{SD}=.78 \mathrm{~s}$ and $M=1.80 \mathrm{~s}, \mathrm{SD}=.83 \mathrm{~s}$, respectively).

\section{Visual attention to the emotional expressions}

To determine whether the groups differed in their visual attention toward the emotional expressions of the actor, two independent sample $t$ tests were performed on the total fixation durations within the AOIs for the fear and happy expression for all frames in which the expression was shown in the video. For both the happy and fear AOIs, there were no group differences in visual attention $(t(38)=-.21, p=.84$ and $t(38)=1.16, p=.26$, respectively $)$. 
Means for total fixation duration within the happy and fear face AOIs are presented in Fig. 3.

\section{Social-emotional calibration: pupil dilation from pre- to post-box}

To determine whether the groups showed social-emotional calibration to the actor, we compared the percentage pupil change from baseline in the pre- and post-box. We conducted two 2 groups (ASD, TD) $\times 2$ boxes (pre, post), repeated measures ANCOVAs, one each for the fear and happy conditions. The dependent variable was mean percentage pupil dilation from baseline, with cognitive ability (MSEL composite score) as a covariate. As the effect of the cognitive ability covariate was not significant for either happy, $F(1,37)=1.25, p=$ $.27, \eta^{2}=.03$, or fear, $F(1,37)=.003, p=.97, \eta^{2}<.001$, both analyses were re-run without this covariate. For both the happy and fear ANOVAs, the group main effect was not significant $\left(F(1,38)<.001, p=.99, \eta^{2}<\right.$ .001 and $F(1,38)=.87, p=.36, \eta^{2}=.02$, respectively), but the box main effect was $(F(1,38)=23.78, p<.001$, $\eta^{2}=.38$ and $F(1,38)=16.90, p<.001, \eta^{2}=.31$, respectively). These effects were both driven by box $\times$ group interactions, with the happy condition significant $\left(F(1,38)=9.42, p=.004, \eta^{2}=.20\right)$ and the fear condition marginally significant $\left(F(1,38)=2.85, p=.09, \eta^{2}=\right.$ .07 , respectively).

Follow-up pairwise comparisons (Bonferroni-corrected) showed that whilst the TD group had a larger mean pupil diameter during the post- vs. pre-box for happy $F(1,38)=30.74, \quad p<.001, \eta^{2}=.45$, and fear $F(1,38)=$
16.82, $p<.001, \eta^{2}=.31$, the ASD group did not show a mean pupil size change of this magnitude in either the happy $F(1,38)=1.45, p=.24, \eta^{2}=.04$ or the fear condition $F(1,38)=2.93, p=.10, \eta^{2}=.07$. Mean percentage pupil dilation from baseline in each condition is presented in Fig. 4, and the difference scores between the pre- and post-box conditions are presented in Fig. 5.

\section{Reaction to actor's emotional expressions}

To examine whether the groups differed in their pupil dilation to the actor's emotional reaction in each of the videos, independent sample $t$ tests were performed on mean percentage pupil dilation from baseline to the emotional reaction of the actor, which was displayed in the video from 18:53 to $25: 31$ in the happy condition and 19:06 to 26:05 in the fear condition. Mean percentage pupil dilation from baseline to the pre-box, emotional reaction and post-box phases of the video are presented in Fig. 6. As the pupil dilation in the post-box may have included prolonged resolution of pupil dilation from the emotional reaction phase, the zoom in section was also included in the figure. Though the group means appeared different on the fear condition, variability within group was large (ASD: $M=8.45, \mathrm{SD}=12.99$; TD: $M=13.32$, $\mathrm{SD}=13.80$ ), so the groups did not differ significantly in their pupil responses to the actor's happy on this condition $(t(38)=1.15, p=.26)$ nor the happy condition $(t(38)=-31, p=.76$; ASD: $M=15.27, \quad \mathrm{SD}=$ 7.44; TD: $M=14.33, \mathrm{SD}=11.15)$.

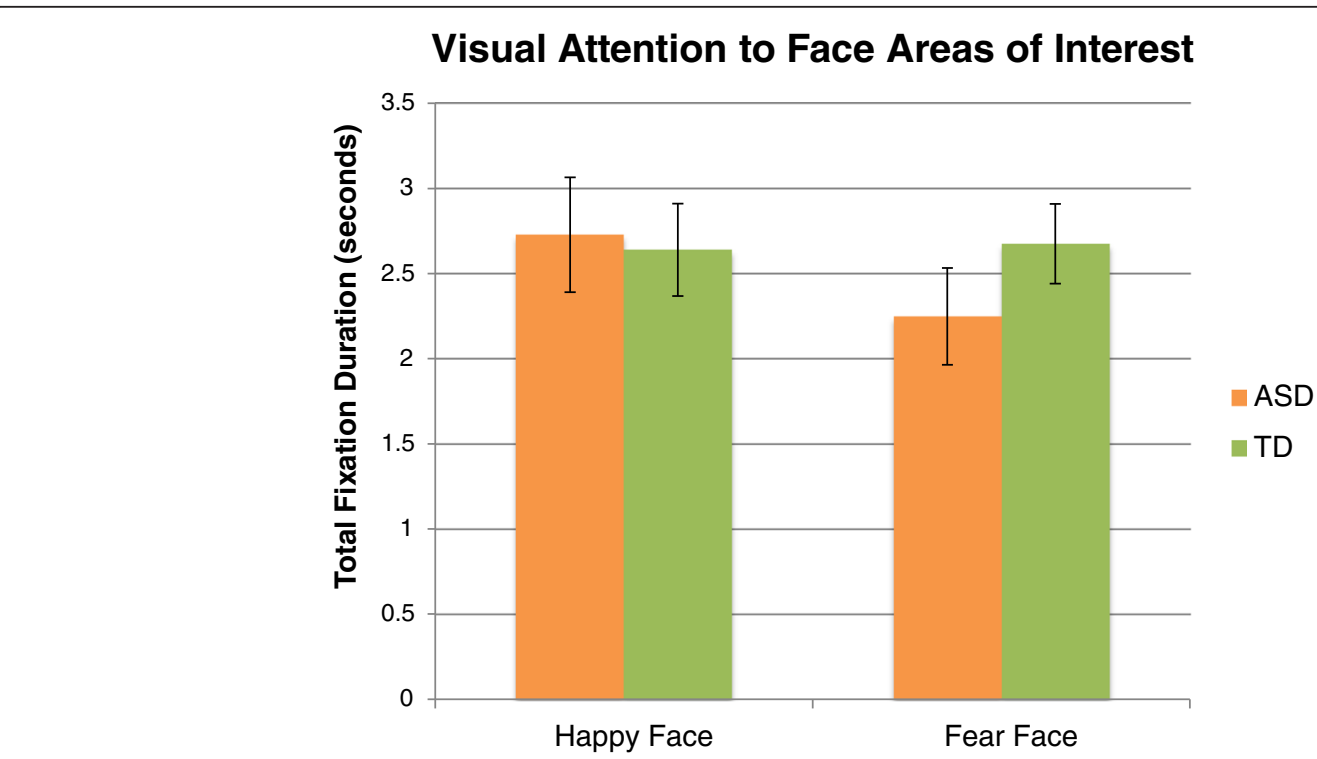

Fig. 3 Visual attention to the emotional expressions. Total fixation duration (sum of individual fixation durations) to the emotional facial expression area of interest for the happy and fear social-emotional calibration videos, for each group. Error bars represent standard error of the mean 


\section{Pupil Dilation: Pre-and Post-Box}

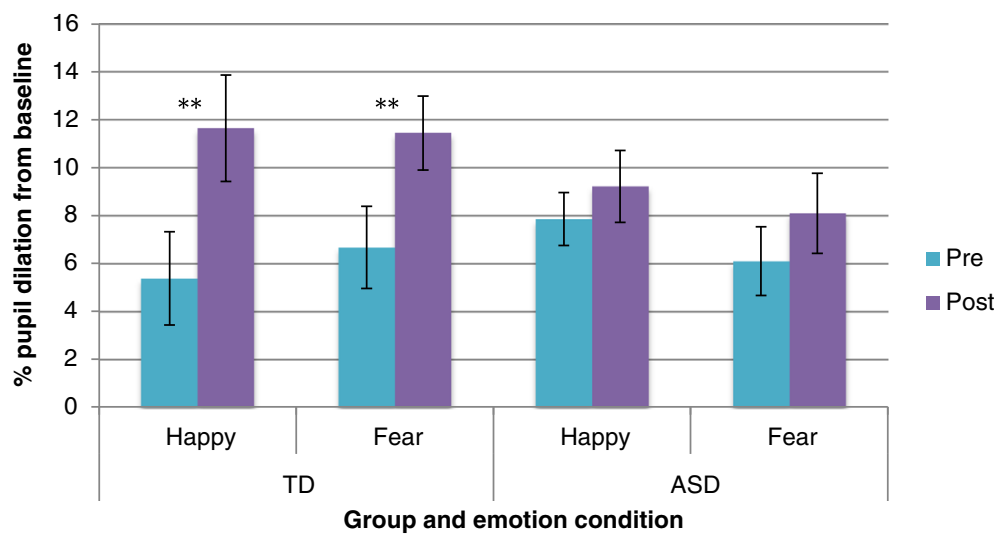

Fig. 4 Percentage dilation from baseline in each emotion condition for the pre- and post-box. Error bars represent standard error of the mean. **Pairwise comparisons (Bonferroni-corrected) show difference pre- to post-box in the TD group only, for happy $\left(p<.001, \eta^{2}=.45\right)$ and fear $\left(p<.001, \eta^{2}=.31\right)$. Error bars represent standard error of the mean

\section{Associations of social-emotional calibration with ASD symptoms}

To explore whether social-emotional calibration was related to ASD symptoms, we ran Pearson correlations between the calibrated ADOS scores (Social Affect scale, Restricted and Repetitive Behaviors scale and Severity Score) and the post-minus pre-box difference score (social-emotional calibration index) on the happy and fear conditions. Positive values index more social-emotional calibration. A moderate negative correlation was found between the Severity Score and the social-emotional calibration index on the happy condition ( $r=-43, p=.03$; see Fig. 7$)$, and there was a trend of negative association between the Restricted and Repetitive Behaviors scale and the social-emotional calibration index on the fear condition $(r=-.36, p=.06)$. No other correlations were significant (Social Affect scale with happy and fear conditions: $r=-.21, p=.19$ and $r=.24, p=.15$, respectively; Restricted and Repetitive Behaviors scale with happy condition: $r=-.29, p=.11$; Severity Score with fear condition: $r=-.11, p=.33$ ).

\section{Discussion}

The aim in this study was to examine whether young children with ASD show calibration to others' emotions.

\section{Pupil Dilation: \\ Pre- to Post-Box Difference Score}

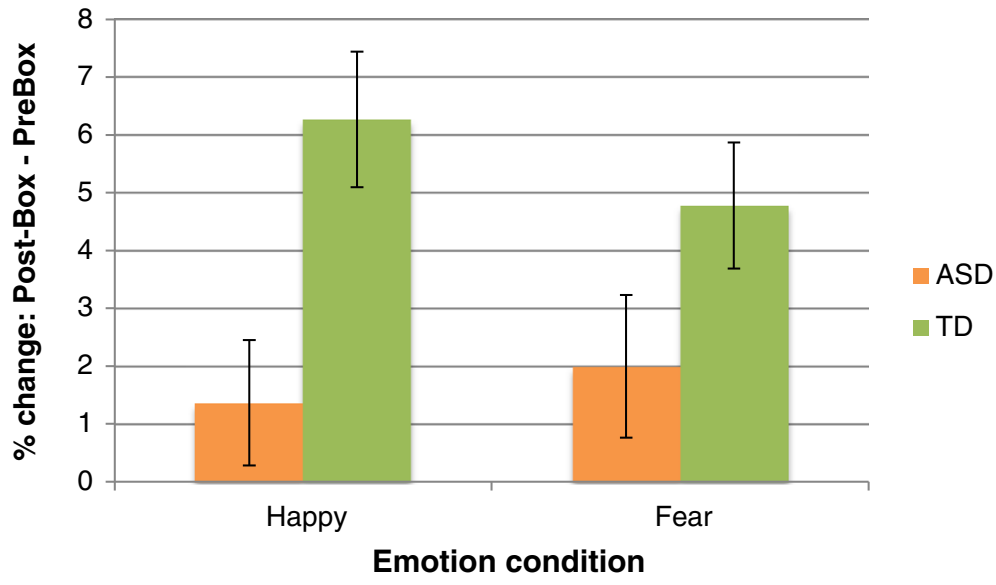

Fig. 5 Post-minus pre-box difference score as an index of social-emotional calibration. Higher scores=more social-emotional calibration. Error bars represent standard error of the mean 


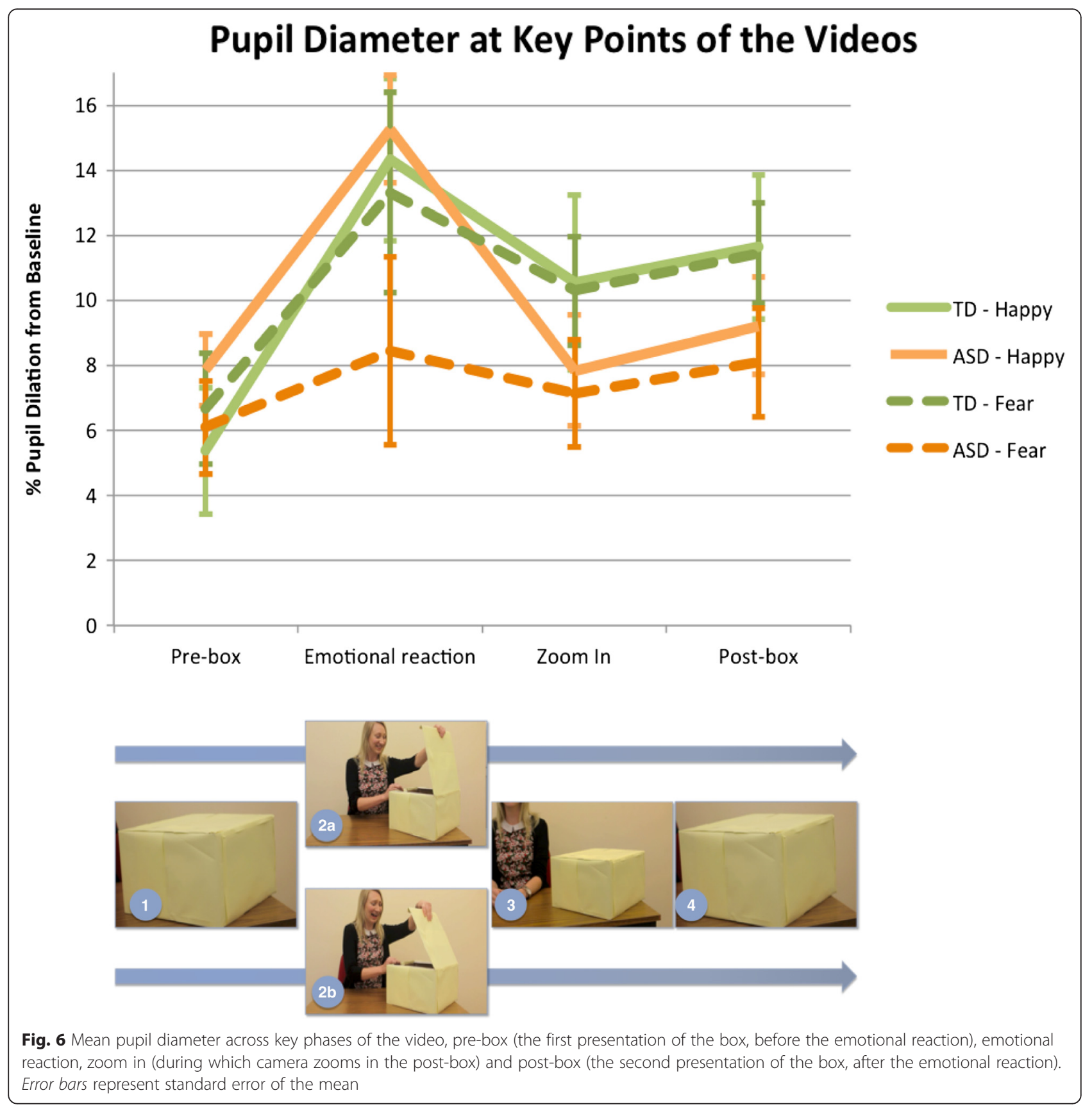

Through the analysis of pupillary reactions, we found that TD children but not children with ASD learned about the emotional value of objects via vicarious emotional reactions. This was not due to a lack of attention or a reduced emotional response to the expressions of the actor; the children with and without ASD did not differ in their visual attention or concurrent pupil dilation to the actor's facial expressions. Thus, results are consistent with the notion of reduced social-emotional calibration in preschoolers with ASD. Interestingly, calibrating to the actor's happy expressions, but not to the fear expressions, was associated with overall ASD severity. This result suggests that autism characteristics are related to the extent to which a person with ASD can quickly internalise and align to the positive emotions of those around them regarding features of their environment. Further research is needed to substantiate this interpretation.

A somewhat surprising finding in the current study was that children with ASD did not differ from their TD peers in their attention to the actor's face. This finding, whilst inconsistent with early eye-tracking research (e.g. [20]), accords with numerous recent findings suggesting normative patterns of visual attention in response to 


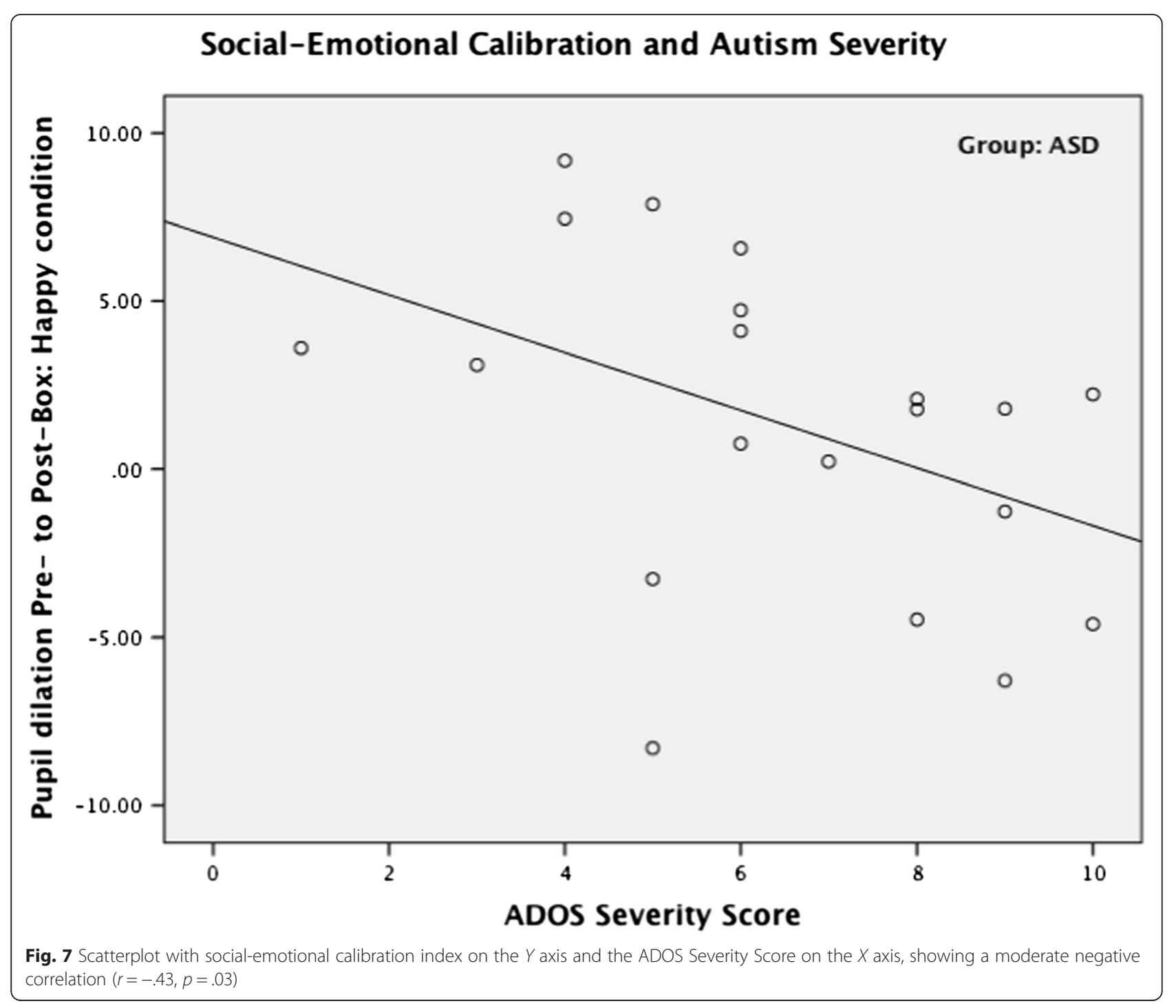

social stimuli in ASD under specific circumstances (e.g. [53, 70-72]). Likewise, reduced concurrent response to the actor's emotions may have been expected given findings of reduced emotional reactivity to others in ASD. However, our finding is consistent with some studies showing no group differences in emotional responses to others during longer presentations of emotions $[3,30]$.

It is important to consider not only both the mechanisms which drive difficulties in social-emotional calibration in ASD but also the effects that difficulties in this area have on other areas of social development and behaviour in ASD. Documented difficulties in dyadic emotional reactivity, emotion recognition and/or in triadic/joint attention in ASD [3, 73-76] are likely to impact social-emotional calibration in this population, and more so than associative learning mechanisms, which are thought to be intact in ASD (e.g. [77]). Further research is now needed to confirm the foundation skills required for social-emotional calibration.

The finding of an association between ASD symptoms and social-emotional calibration may help to explain the idiosyncratic responses individuals with this disorder characteristically have to people and objects. Individuals with ASD also commonly present with idiosyncratic emotional reactions to sensory features of the environment; one hypothesis is that these arise from difficulties calibrating to others' emotional states about these sensations, i.e. to the neutral emotional states during everyday activities. Common everyday activities can cause extreme emotional reactions to children with ASD (e.g. vacuuming, having a haircut, rearranging home furniture and doing the grocery shopping), leading to meltdowns and impairing family functioning [7, 78]. Thus, future research should examine in more detail the relationship between social-emotional calibration and atypical emotional 
reactions to sensory features of the environment in ASD and between social-emotional calibration and emotion regulation in this population. This result has important implications for intervention strategies and targets, providing a case for using therapeutic styles which capitalise on positive-affect joint attention frameworks for teaching skills [79-81] and explicitly teaching social-emotional calibration to positive events, objects and other experiences.

A deficit in social-emotional calibration arguably has important implications for social and cultural development. Aligning to others' emotions in a myriad of contexts, throughout development, teaches children about their world and the objects, events and people in it, including the cultural practices, habits and attitudes of a particular society. These everyday learning opportunities provide children with rich information to guide their behaviour, judgements and attitudes. A deficit in calibrating to others' emotion/s regarding features of the environment, as documented in this study in children with ASD, is likely to negatively impact learning from their social environment and their participation in the cultural and sub-cultural practices of the societies in which they live.

As social-emotional calibration may be characterised as an implicit learning process, difficulties in calibrating to others' emotions may be a result of difficulties in implicit learning in ASD and so this matter should be investigated further. Furthermore, as the paradigm in the current study was essentially a one-trial learning paradigm, further work is needed to understand whether people with ASD do calibrate to others' emotions about features of the environment following multiple presentations. Other factors which affect social learning (such as temperament and social closeness to the target person) are likely to have an impact on social-emotional calibration and should also be investigated in future work. In addition to physiological reactivity indices of socialemotional calibration, another future direction would be to investigate changes in behavioural patterns that may occur after calibrating to one's emotions.

\section{Limitations}

Whilst the inclusion of an age-matched TD group afforded an understanding of normative reactivity, it is important to acknowledge that the two groups were not matched on cognitive ability. The inclusion of a chronological- and mental age-matched group would have been ideal, and further research should seek to incorporate such a control group. Nonetheless, it should be noted that the cognitive ability covariate was not significant in the analysis.

As the children with ASD in the current study were mostly low functioning with significantly delayed receptive language ability, it was not possible to administer an emotion recognition task to understand the contribution of emotion recognition difficulties to diminished socialemotional calibration. Future research should aim to include such a task, only possible with more able children, to clarify this issue.

Although physiological measures can provide insight into the emotion processing of lower-functioning individuals, physiological measures do not distinguish valence of one's emotional reaction (i.e. whether viewing happy expressions makes oneself feel happy), rather they provide information on emotional arousal. Therefore, future research on social-emotional calibration should incorporate measures, such as behavioural coding of facial affect, to provide information on the valence of emotional reactions and how these relate to aligning with others' behaviour.

\section{Conclusions}

The current study explored whether children with ASD calibrate to others' emotional expressions about features of their environment, an aspect of social learning in ASD that has received little empirical attention to date. The pupil dilation results are consistent with the presence of social-emotional calibration in TD children, but not in children with ASD. By studying this process of socialemotional calibration, we can better understand the difficulties people with ASD have in learning from others and participating in cultural and sub-cultural practices, as well as the idiosyncratic responses that are characteristic of this population. Further work is needed to substantiate this interpretation and understand the mechanisms that underlie social-emotional calibration and the effects of this phenomenon on social development.

\section{Abbreviations}

ADOS, Autism Diagnostic Observation Schedule; AOl, area of interest; ASD, autism spectrum disorder; ECG, electrocardiogram; ERP, event-related potentials; fMRl, functional magnetic resonance imaging; MSEL, Mullen Scales of Early Learning; TD, typically developing

\section{Acknowledgements}

First and foremost, the authors would like to thank the families who took part in this study. The authors are also extremely grateful to the wonderful staff at the La Trobe University Children's Centre and Victorian Autism Specific Early Learning and Care Centre (especially Jenny Reynolds, Ed Duncan, Jess Feary, Kristy Capes, Dianna Pell, Shannon Upson, Sue Schoene, Danni Dart and Sanji Ahsan). We would also like to acknowledge Russell Beaton for his ingenious work on programming for the data reduction and the actor, CB, who generously gave her time to make the stimuli videos (her written consent for the publication of pictures from these videos is held by the authors' institution, available for review by the Editor-in-Chief if necessary). Lastly, the authors are grateful to John Herrington and Ben Yerys from the Center for Autism Research, Children's Hospital of Philadelphia, whose feedback encouraged the first author to refine concepts and terminology discussed in the manuscript.

\section{Authors' contributions}

$\mathrm{HN}$ and GV conceived the study and design. HN conducted the research testing, arranged for the pupillary data reduction and analysed the data, with guidance from GV and CD. HN, GV and CD were involved in the interpretation of the data. HN drafted the manuscript, and GV and CD revised it critically, giving important intellectual content. All authors read and approved the final manuscript. 


\section{Competing interests}

The authors declare that they have no competing interests.

\section{Consent for publication}

Written consent has been obtained from the person featured in Fig. 1 to publish her picture in the manuscript.

\section{Ethics approval and consent to participate}

The research was approved by the La Trobe University Human Ethics Committee (approval number 11-052), and consent from the children's parents was obtained according to the Declaration of Helsinki (BMJ 1991; 302: 1194).

\section{Author details}

${ }^{1}$ Center for Mental Health Policy and Services Research, Perelman School of Medicine, Department of Psychiatry, University of Pennsylvania, Philadelphia, PA 19104, USA. ${ }^{2}$ Olga Tennison Autism Research Centre, School of Psychological Science, La Trobe University, Melbourne, Victoria 3086, Australia. ${ }^{3}$ A. J. Drexel Autism Institute, Drexel University, Philadelphia, PA 19104, USA.

Received: 25 May 2016 Accepted: 28 July 2016

Published online: 30 August 2016

\section{References}

1. American Psychiatric Association [APA]. Diagnostic and statistical manual of mental disorders, fifth edition [DSM-5]. Arlington: American Psychiatric Association; 2013.

2. Hobson RP. Autism and emotion. In: Handbook of autism and pervasive developmental disorders, fourth edition. 2014

3. Nuske HJ, Vivanti G, Dissanayake C. Are emotion impairments unique to, universal, or specific in autism spectrum disorder? A comprehensive review. Cogn Emot. 2013;27:1042-61.

4. Lord C, Rutter M, Le Couteur A. Autism diagnostic interview-revised: a revised version of a diagnostic interview for caregivers of individuals with possible pervasive developmental disorders. J Autism Dev Disord. 1994;24:659-85.

5. Mazefsky CA, Herrington J, Siegel M, Scarpa A, Maddox BB, Scahill L, White SW. The role of emotion regulation in autism spectrum disorder. J Am Acad Child Adolesc Psychiatry. 2013;52:679-688.

6. Schaaf RC, Miller $\amalg$, Seawell D, O'Keefe S. Children with disturbances in sensory processing: a pilot study examining the role of the parasympathetic nervous system. Am J Occup Ther. 2003;57:442.

7. Colvin G, Sheehan MR. Managing the cycle of meltdowns for students with autism spectrum disorder. New York: Corwin Press; 2012.

8. Campos J, Stenberg C. Perception, appraisal, and emotion: the onset of social referencing. In: Lamb ME, Sherrod LR, editors. Infant social cognition: empirical and theoretical considerations. Hillsdale: Erlbaum; 1981. p. 273-314.

9. Feinman S. Social referencing in infancy. Merrill-Palmer Q. 1982:28:445-470.

10. Hornik R, Risenhoover N, Gunnar M. The effects of maternal positive, neutral, and negative affective communications on infant responses to new toys. Child Dev. 1987;58:937-944.

11. Mumme DL, Fernald A, Herrera C. Infants' responses to facial and vocal emotional signals in a social referencing paradigm. Child Dev. 1996;67:3219-37.

12. Sorce J, Emde R, Campos J, Klinnert M. Maternal emotional signaling: its effect on the visual cliff behavior of 1-year-olds. Dev Psychol. 1985;21:195-200.

13. Trevarthen C. First things first: infants make good use of the sympathetic rhythm of imitation, without reason or language. J Child Psychother. 2005;31:91-113.

14. Young N, Hudry K, Trembath D, Vivanti G. Information seeking in a pedagogical context by children with autism spectrum disorders and children with developmental delays. Am J Intellect Dev Disabil. 2015;39:231-41.

15. Sigman MD, Kasari C, Kwon JH, Yirmiya N. Responses to the negative emotions of others by autistic, mentally retarded, and normal children. Child Dev. 1992;63:796-807.

16. Vivanti G, Rogers SJ. Autism and the mirror neuron system: insights from learning and teaching. Philos Trans R Soc Lond B Biol Sci. 2014;369:20130184.

17. Ahearn WH, Parry-Cruwys D, Toran T, MacDonald J. Stimulus salience in autism: a social learning disorder. In: Autism Service Delivery. New York: Springer; 2015:75-111.

18. Mundy P, Mastergeorge A, Mclntyre N. Effects of autism on social learning and social attention. In: Educational interventions for students with autism. San Francisco: John Wiley \& Sons; 2012.
19. Jones W, Klin A. Attention to eyes is present but in decline in 2-6-monthold infants later diagnosed with autism. Nature. 2013:504:427-31.

20. Klin A, Jones W, Schultz R, Volkmar F, Cohen D. Visual fixation patterns during viewing of naturalistic social situations as predictors of social competence in individuals with autism. Arch Gen Psychiatry. 2002;59:809-16.

21. Hatfield E, Cacioppo JT, Rapson RL. Emotional contagion. Cambridge: Cambridge university press; 1994.

22. Puce A, Bertenthal BI. The many faces of social attention. 2015.

23. Blair R. Psychophysiological responsiveness to the distress of others in children with autism. Personal Individ Differ. 1999;26:477-85.

24. Kuchinke L, Schneider D, Kotz SA, Jacobs AM. Spontaneous but not explicit processing of positive sentences impaired in Asperger's syndrome: pupillometric evidence. Neuropsychologia. 2011;49:331-8.

25. Hubert BE, Wicker B, Monfardini E, Deruelle C. Electrodermal reactivity to emotion processing in adults with autistic spectrum disorders. Autism. 2009:13:9-19.

26. Wagner JB, Hirsch SB, Vogel-Farley VK, Redcay E, Nelson CA. Eye-tracking, autonomic, and electrophysiological correlates of emotional face processing in adolescents with autism spectrum disorder. J Autism Dev Disord. 2012;43: 188-99.

27. Baron-Cohen S, Ring HA, Wheelwright S, Bullmore ET, Brammer MJ, Simmons A, Williams SCR. Social intelligence in the normal and autistic brain: an fMRI study. Eur J Neurosci. 1999;11:1891-8.

28. Critchley HD, Daly EM, Bullmore E, Williams SCR, Van Amelsvoort T, Robertson DM, Rowe A, Phillips M, McAlonan G, Howlin P, Murphy DGM. The functional neuroanatomy of social behaviour. Brain. 2000;123:2203-12.

29. Kleinhans NM, Richards T, Weaver K, Johnson LC, Greenson J, Dawson G, Aylward E. Association between amygdala response to emotional faces and social anxiety in autism spectrum disorders. Neuropsychologia. 2010;48:3665-70.

30. Nuske HJ, Vivanti G, Hudry K, Dissanayake C. Pupillometry reveals reduced unconscious emotional reactivity in autism. Biol Psychol. 2014;101:24-35.

31. Akechi H, Senju A, Kikuchi $Y$, Tojo Y, Osanai H, Hasegawa T. Does gaze direction modulate facial expression processing in children with autism spectrum disorder? Child Dev. 2009;80:1134-46.

32. Bal E, Harden E, Lamb D, Van Hecke AV, Denver JW, Porges SW. Emotion recognition in children with autism spectrum disorders: relations to eye gaze and autonomic state. J Autism Dev Disord. 2010:40:358-70.

33. Korpilahti P, Jansson-Verkasalo E, Mattila M-L, Kuusikko S, Suominen K, Rytky S. Pauls DL, Moilanen I. Processing of affective speech prosody is impaired in Asperger syndrome. J Autism Dev Disord. 2007;37:1539-49.

34. Oberman LM, Winkielman P, Ramachandran VS. Slow echo: facial EMG evidence for the delay of spontaneous, but not voluntary, emotional mimicry in children with autism spectrum disorders. Dev Sci. 2009;12:510-20.

35. Webb SJ, Dawson G, Bernier R, Panagiotides H. ERP evidence of atypical face processing in young children with autism. J Autism Dev Disord. 2006:36:881-90.

36. Bradley MM, Miccoli L, Escrig MA, Lang PJ. The pupil as a measure of emotional arousal and autonomic activation. Psychophysiology. 2008;45:602-7.

37. Partala T, Surakka V. Pupil size variation as an indication of affective processing. Int J Hum-Comput Stud. 2003:59:185-98

38. Kret ME. Emotional expressions beyond facial muscle actions. A call for studying autonomic signals and their impact on social perception. Front Psychol. 2015;6:article 711.

39. Bradshaw J. Pupil size as a measure of arousal during information processing. Nature. 1967;216:515-6.

40. Silk JS, Dahl RE, Ryan ND, Forbes EE, Axelson DA, Birmaher B, Siegle GJ. Pupillary reactivity to emotional information in child and adolescent depression: links to clinical and ecological measures. Am J Psychiatry. 2007;164:1873-80.

41. Siegle GJ, Granholm E, Ingram RE, Matt GE. Pupillary and reaction time measures of sustained processing of negative information in depression. Biol Psychiatry. 2001;49:624-36.

42. Felmingham KL, Rennie C, Manor B, Bryant RA. Eye tracking and physiological reactivity to threatening stimuli in posttraumatic stress disorder. J Anxiety Disord. 2011;25:668-73.

43. Burkhouse KL, Siegle GJ, Gibb BE. Pupillary reactivity to emotional stimuli in children of depressed and anxious mothers. J Child Psychol Psychiatry. 2014;55:1009-16.

44. Franzen PL, Buysse DJ, Dahl RE, Thompson W, Siegle GJ. Sleep deprivation alters pupillary reactivity to emotional stimuli in healthy young adults. Biol Psychol. 2009;80:300-5.

45. Anderson C, Colombo J, Shaddy DJ. Visual scanning and pupillary responses in young children with autism spectrum disorder. J Clin Exp Neuropsychol. 2006;28:1238-56. 
46. Nuske HJ, Vivanti G, Dissanayake C. Brief report: evidence for normative resting-state physiology in autism. J Autism Dev Disord. 2014;44:2057-63.

47. Anderson CJ, Colombo J. Larger tonic pupil size in young children with autism spectrum disorder. Dev Psychobiol. 2009;51:207-11.

48. Anderson CJ, Colombo J, Unruh KE. Pupil and salivary indicators of autonomic dysfunction in autism spectrum disorder. Dev Psychobiol. 2012; 55:465-82.

49. Blaser E, Eglington L, Carter AS, Kaldy Z. Pupillometry reveals a mechanism for the autism spectrum disorder (ASD) advantage in visual tasks. Sci Rep. 2014:4:article 4301

50. Nyström P, Gredebäck G, Bölte S, Falck-Ytter T. Hypersensitive pupillary light reflex in infants at risk for autism. Mol Autism. 2015;6:1.

51. Nuske HJ, Vivanti G, Dissanayake C. No evidence of emotional dysregulation or aversion to mutual gaze in preschoolers with autism spectrum disorder: an eye-tracking pupillometry study. J Autism Dev Disord. 2015;45:3433-45.

52. Falck-Ytter T. Face inversion effects in autism: a combined looking time and pupillometric study. Autism Res. 2008;1:297-306.

53. Nuske HJ, Vivanti G, Dissanayake C. Reactivity to fearful expressions of familiar and unfamiliar people in children with autism: an eye-tracking pupillometry study. J Neurodev Disord. 2014;6:1-16.

54. Krach S, Kamp-Becker I, Einhäuser W, Sommer J, Frässle S, Jansen A, Rademacher L, Müller-Pinzler L, Gazzola V, Paulus FM. Evidence from pupillometry and fMRI indicates reduced neural response during vicarious social pain but not physical pain in autism. Hum Brain Mapp. 2015;36:4730-44.

55. Sepeta L, Tsuchiya N, Davies MS, Sigman M, Bookheimer SY, Dapretto M. Abnormal social reward processing in autism as indexed by pupillary responses to happy faces. J Neurodev Disord. 2012;4:17.

56. Mullen EM. Mullen scales of early learning. Circle Pines: AGS; 1995

57. Dykens EM, Lense M. Intellectual disabilities and autism spectrum disorder: a cautionary note. In: Amaral D, Dawson G, Geschwind DH, editors. Autism spectrum disorders. Oxford: Oxford University Press; 2011.

58. Joseph RM. The significance of IQ and differential cognitive abilities for understanding ASD. In: Fein D, editor. The neuropsychology of autism. 2011. p. 281-94.

59. Lord C, Rutter M, DiLavore PC, Risi S. Autism diagnostic observation schedule-WPS (ADOS-WPS). Los Angeles: Western Psychological Services; 1999.

60. Gotham K, Risi S, Pickles A, Lord C. The autism diagnostic observation schedule: revised algorithms for improved diagnostic validity. J Autism Dev Disord. 2007;37:613-27.

61. Janisse MP. Pupillometry: the psychology of the pupillary response. Washington, DC: Hemisphere Publishing Corporation; 1977.

62. Farzin F, Rivera SM, Hessl D. Brief report-visual processing of faces in individuals with fragile $x$ syndrome: an eye tracking study. J Autism Dev Disord. 2009;39:946-52

63. Hepach R, Vaish A, Tomasello M. Young children are intrinsically motivated to see others helped. Psychol Sci. 2012;23:967-72.

64. Chau M, Betke M. Real time eye tracking and blink detection with USB cameras. Technical Report. Boston: Boston University Computer Science Department; 2005

65. Martineau J, Hernandez N, Hiebel L, Roché L, Metzger A, Bonnet-Brilhault F. Can pupil size and pupil responses during visual scanning contribute to the diagnosis of autism spectrum disorder in children? J Psychiatr Res. 2011;45:1077-82.

66. Bergamin $\mathrm{O}$, Kardon $\mathrm{RH}$. Latency of the pupil light reflex: sample rate, stimulus intensity, and variation in normal subjects. Investig Opthalmology Vis Sci. 2003:44:1546.

67. Komogortsev OV, Gobert DV, Jayarathna S, Gowda SM. Standardization of automated analyses of oculomotor fixation and saccadic behaviors. Biomed Eng IEEE Trans On. 2010;57:2635-45.

68. Poole A, Ball $\sqcup$. Eye tracking in human-computer interaction and usability research: current status and future prospects. In: Ghaoui $C$, editor. Encyclopedia of human computer interaction. Hershey, PA: Idea Group: 2006. p. 211-9.

69. Tabachnick BG, Fidell LS. Using multiple statistics. New York, NY: Harper Collins Publishers; 1996.

70. Chawarska K, Macari S, Shic F. Context modulates attention to social scenes in toddlers with autism. J Child Psychol Psychiatry. 2012;53:903-13.

71. Falck-Ytter T, Von Hofsten C. How special is social looking in ASD? A review. Prog Brain Res. 2011;189:209-22.
72. Vivanti G, Dissanayake C. Propensity to imitate in autism is not modulated by the model's gaze direction: an eye-tracking study. Autism Res. 2014:7:392-9.

73. Mundy P. Joint attention and social-emotional approach behavior in children with autism. Dev Psychopathol. 1995;7:63-82.

74. Charman T, Swettenham J, Baron-Cohen S, Cox A, Baird G, Drew A. Infants with autism: an investigation of empathy, pretend play, joint attention, and imitation. Dev Psychol. 1997;33:781.

75. Dawson G, Toth K, Abbott R, Osterling J, Munson J, Estes A, Liaw J. Early social attention impairments in autism: social orienting, joint attention, and attention to distress. Dev Psychol. 2004;40:271

76. Mundy P, Sullivan L, Mastergeorge AM. A parallel and distributed processing model of joint attention, social-cognition and autism. Autism Res Off J Int Soc Autism Res. 2009;2:2-21.

77. Williams DL, Goldstein G, Minshew NJ. Neuropsychologic functioning in children with autism: further evidence for disordered complex informationprocessing. Child Neuropsychol. 2006;12:279-98.

78. Schaaf RC, Toth-Cohen S, Johnson SL, Outten G, Benevides TW. The everyday routines of families of children with autism: examining the impact of sensory processing difficulties on the family. Autism. 2011;15:373-389.

79. Rogers S, Dawson G. Early start denver model for young children with autism: promoting language, learning, and engagement. New York: Guilford Press; 2010.

80. Schreibman L, Dawson G, Stahmer AC, Landa R, Rogers SJ, McGee GG, Kasari C, Ingersoll B, Kaiser AP, Bruinsma Y, et al. Naturalistic developmental behavioral interventions: empirically validated treatments for autism spectrum disorder. J Autism Dev Disord. 2015:45:2411-28.

81. Kasari C, Freeman S, Paparella T. Joint attention and symbolic play in young children with autism: a randomized controlled intervention study. J Child Psychol Psychiatry. 2006;47:611-20.

\section{Submit your next manuscript to BioMed Central and we will help you at every step:}

- We accept pre-submission inquiries

- Our selector tool helps you to find the most relevant journal

- We provide round the clock customer support

- Convenient online submission

- Thorough peer review

- Inclusion in PubMed and all major indexing services

- Maximum visibility for your research

Submit your manuscript at www biomedcentral.com/submit
Ciomed Central 